\title{
Confucian Free Energy: The Predictive Mind in Ancient China
}

\author{
Peter Thestrup Waade
}

March 10th, 2020 


\begin{abstract}
Roger Ames presents an interpretation of the classic Confucian philosophy where the world is seen as an always ongoing, radically interrelational, holistic process best described in terms of focus and field. Without any permanent ground truth, its inhabitants must be action-oriented way-makers: irreducibly social optimizers, and contextual co-creators, of their shared cosmos. Meanwhile, a pragmatic turn is happening in the cognitive sciences, instantiated in a new and ambitious theoretical framework: Karl Friston' s free energy principle, which aims to be a unifying paradigm for the sciences of brain, life and mind. Based on first principles from statistical physics, it describes humans - and self-organizing systems in general - as continually modelling and predicting the world. The full spectrum of human cognition and behavior is then generated by a single mechanism: the minimization of the difference between predicted and actual sensory inputs. Although still controversial, the free energy principle is gaining traction within robotics, biology, neuroscience, psychology, social sciences and philosophy of mind. I here compare these two worldviews from so vastly different backgrounds, and find, perhaps surprisingly, that they in many respects are immediately compatible. Social co-creativity across spatio-temporal scales, contextualization, action-orientation and the Confucian virtues naturally translate to or integrate with free energy principle terms. Even when in seeming disagreement, for example on the question of the appearance/reality dichotomy, or that of reductive materialism which often separates the sciences and humanities in general, the two frameworks seem able to positively inform or nuance each other. This multidisciplinary comparison is interesting because it can potentially inform or guide the two theories, and address recent critiques of Ames, and as a proof of concept that bridges can be built across times, cultures and academic traditions.
\end{abstract}

page 2 of 22 


\section{Introduction}

In 2019, Edward Slingerland published his book Mind and body in Ancient China: Beyond Orientialism and the Myth of Holism (Slingerland, 2019a) in which he criticizes Roger Ames' interpretation of Confucian thought. Ames is attempting to interpret classical Confucianism on its own terms as opposed to the historically prevalent eurocentric perspectives which attempt to insert Confucianism within the Western philosophical and theological tradition (Ames, 2011). Slingerland, on the other hand, claims that Ames and related scholars are "strong social constructivists" who put too little weight on biologically founded human universals when interpreting the Confucian texts. The main critique in Slingerland's book was against Ames' and related scholars' characterization of the Confucian worldview as holistic, asserting that the ancient Chinese could not have thought of mind and body (and other Western dualistic pairs like reality/appearance) as inseparable parts of a continuum. ${ }^{1}$ One part of Slingerland's argument is based on a classic approach within the humanities, another in digital, automated text analysis methods, and a third in theoretical work from cognitive science. In this paper I do not evaluate Slingerland' s classical philosophy and digital humanities approaches. It should be noted, though, that there has been a correspondence between James Behuniak and Slingerland (Behuniak, 2019b; Slingerland, 2019b; Behuniak, 2019a), where Behuniak claims that Slingerland is attributing to Ames and his related scholars an extreme version of their views in order to refute them - a classic strawman argument. Instead, I focus on his claim that the best insights in cognitive science indicate that weak mind/body dualism is a human universal. I contradict this point based on a growing and influential theoretical framework within cognitive science, the free energy principle (FEP), and show that it in many ways is compatible with Ames' Confucianism. This compatibility means that cognitive science does not unequivocally support Slingerland's claims.

Within cognitive science and its related academic fields, including psychology, neuroscience, robotics and philosophy of mind, there is an ongoing turn away from representational and towards enactivist theories. Where before the mind was thought of as a passive observer attempting to create an optimal mental representation of the world, it is instead described as an active agent that participates dynamically in creating its own experience of the world, and to whom cognition and mental activity always subserves action (Engel et al., 2016). The FEP lies at the heart of this possibly paradigmatic turn. It is an ambitious and controversial attempt at a unified theoretical framework for biological and human behaviour and cognition, developed by Karl Friston (Friston et al., 2006; Friston and Stephan, 2007; Friston, 2010). The framework describes living systems as continually modelling and predicting the world, and claims that both human behavior and cognition can be understood as essentially a prediction error minimization (PEM) mechanism (Hohwy, 2013). At its most radical level, however, not only humans but any self-organizing system that actively resists entropy must through action and perception reduce the discrepancies between its predicted

\footnotetext{
${ }^{1}$ See also for example (Slingerland and Chudek, 2011; Slingerland, 2013) for earlier work on the same topic
} 
and received sensory inputs (Ramstead et al., 2018). The FEP is based in first principles from statistical physics and information theory, and is mathematically implementable; it is as such both mechanical and materialist (Hohwy, 2013). However, since it aims to create a unified account of the mind, the theory must be able to communicate with other theories about how humans behavior and experience, across academic field. The worldview of classical Confucianism is one such theory, which comes out of not only a different academic tradition, but a different culture and time. The comparison is mutually beneficial. For the FEP, it provides inspiration and direction for future work, and a different vocabulary that may help describe the materialist theory in terms more compatible with our intuitively lived experiences. At the same time, the FEP might provide nuance to Confucian ideas, or even allow for their empirical testing. The compatibility of the two theories also provides perspective to the strand of Slingerland's critique that is based in cognitive science. As such, the comparison can contribute to each field separately, but can ultimately be part a larger movement towards bridging the humanities and sciences.

In this paper I will first give an overview of the Confucian worldview, as it is laid out by Roger Ames, and give a conceptual introduction to FEP. I will then describe where I think the two frameworks are naturally compatible, and explore where they might disagree or complement each other.

\section{The Confucian World}

In the following section, I give a brief overview of some main tenets in classic Confucianism. This interpretation of the philosophy is entirely based on work by Roger Ames (Ames, 2011), and so represents the views criticized by Edward Slingerland (Slingerland, 2019a). It is this interpretation of Confucianism which I will compare with the free energy principle (FEP) in sections 4 and 5.

\subsection{A $q i$ 气 cosmology}

The ancient Chinese metaphysics, perhaps most clearly presented in the Yijing 易经, is one of process: the cosmos does not consist of static things that are separate in all but external relations, but is rather a continuous flow of interwoven happenings. A person is not an ontological substance which performs actions; instead, he is a living, dynamic process that equally includes all he is and does. There are not things but events, and not a (or the) physical world which exists; rather worlding happens. Thus, everything is always changing, and to understand the world is to understand those changes, as opposed to a platonic striving towards permanent knowledge of abstract concepts. In other words: what is real is simply that which happens, not a being but a constant becoming. This lack of a permanent underlying substance means that categories are defined paranomastically, by similarity, rather than by necessary characteristics. In the process ontology, no two events are ever completely separate, but always implies each other. Anything in the cosmos exists entirely at the pleasure of, indeed as part of, everything else. This radical relationality is what makes the Confucian 
worldview holistic, for when everything makes up a continuous whole, parts cannot truly exclude each other. Instead of the dualistic mind/body and reality/appearance dichotomies in classic Greek philosophy, the ancient Chinese saw mind and body as complementary yin yang 阴阳 poles of a single continuum. Mind and body, different people, and indeed any other kind of apparent separation come not from ontologically different substances, but are abstractions out of reality. This is the theme when Mencius famously writes that " all the ten-thousand events are implicated here in me" ${ }^{2}$ suggesting that the entire cosmos is implied within him, and is as much part of him as he is part of it. How, then, do we understand a mind, a self? Here Ames refers to the Daoist story of Zhuangzi walking on the bridge over the river Hao. Zhuangzi knows about happiness only from the place he stands - that is, he is a specific perspective within the holistic cosmos that is in no way separate from it. To describe this inseparability of the one and the many yiduobufen一多不分, Ames often applies the language of focus and field. Here, a mind, self or person is a focused perspective within the endless field that is the cosmos, and while each focus is entirely unique, there are no sharp boundaries between it and everything else (Ames, 2011).

\subsection{Participating in the world}

The Confucian project is one of becoming (rather than being born as) consummately human. For the irreducibly social human, this process of self-creation is simultaneously a process of creating others and society in general, for we as humans are irreducibly social. This is clear from the virtues within the Confucian project. To defer to roles and social grammar $l i$ 礼, and to be trustworthy in conduct $x i n$ 信, are inherently social concepts, and even to act optimally appropriately yi 义 should be understood within a socio-cultural context. Even the character for being consummately human ren 仁 is a combination of the characters for "person" ren 人 and "two" er 二, indicating that it is only possible to be fully human in the interplay with others. The Confucian project also calls explicitly for action above knowledge, or rather, for knowledge as bound within action. The word for "knowing" $z h i$ 智 is perhaps better translated as "realizing", carrying simultaneously the sense of understanding and creating. This focus on action also suits the lack of a substance ontology - in a cosmos that is process, knowledge is only useful as long as it can be used for active participation in the changing world and society. Confucianists are not interested in finding the Truth - rather, they are looking for a way to optimally exist in the world. This means that knowledge is always contextual and personal, where sometimes even diametrically opposite answers are appropriate for the same question posed by different people. Confucianism also emphasizes an internalization of values, rather than simply following external motivations for acting properly in society. The exemplary person junzi 君子 cares for the root of her person to affect her behaviour. Then acting appropriately becomes not only easy and spontaneous ziran 自然 like water gushing through a hole in river dam, but also pervasive, happening naturally across all contexts, both when we are alone and with others. The focus on

\footnotetext{
${ }^{2}$ All quotes from Mencius are taken from Ames, translation in progress
} 
participating in the world is perhaps most evident in the Confucian ideal person - the Sage shengren 圣人. That is the person who through understanding the changes in the world affects it profoundly and pervasively, ultimately partaking in shaping the entire cosmos by bringing the human experience together like a symphony, enchanting it and making it meaningful. Being able to affect the world so pervasively is what Mencius calls having a " flood-like qi" haoran zhi qi 浩然之气 (Ames, 2011).

\section{The Free Energy Principle}

\subsection{The brain as a hypothesis tester}

The free energy principle (FEP) is an influential theoretical framework for describing brain, behavior, and the dynamics of self-organizing systems, developed by Karl Friston (Friston et al., 2006, 2007, 2010). The theory is immensely ambitious, attempting, perhaps for the first time ever (Hohwy, 2013), to establish a grand theory which is at the same time mathematically describable, neuro-scientifically implementable, and can account for the entire spectrum of cognitive and behavioral processes using only a single, simple mechanism: prediction error minimization (PEM). The theory generalizes, formalizes or subsumes many other earlier theories, like Bayesian brain, predictive processing, explore/exploit paradigms, reinforcement learning, and embodiment theories, and it enters in a variety of discussions from biology to philosophy of mind. It was originally designed to describe the brain, but recently its scope has increased to life and self-organizing systems in general, from inter-cellular processes to cultural dynamics. It is this broad universality which allows for comparison with equally broad philosophical stances, like Confucianism. Of course, it is controversial whether the theory will live up to this ambitious project, and the value of unified grand theories in general is debated (Colombo and Wright, 2017). There exist less radical stances, where the principle is only applied to the brain, and where PEM is only one brain process among others rather than the only generative mechanism (Hohwy, 2013). I will not evaluate these criticisms here, but will in the interest of philosophical comparison use the radical and general version of the FEP. I am also not able to here evaluate the large amount of underlying technical, mathematical and neuroscientific work, and will in general not go into the technical aspects at all except where they are relevant for the larger conceptual discussion. ${ }^{3}$

In the FEP, the brain is essentially a hypothesis-generating and -testing mechanism, that actively attempts to gather information about the world in order to create continuously better hypotheses about its states, so as to be able to act optimally. This idea is not new; it goes back at least to the ideas of Helmholtz ${ }^{4}$, which was formulated as a direct response to Kant' $\mathrm{s}$ ideas ${ }^{5}$ that the mind contributes to the shaping of experience (Hohwy, 2013). The difference is that it now has

\footnotetext{
${ }^{3}$ If interested in technical and mathematical aspects, see for example (Friston, 2010; Buckley et al., 2017; Bogacz, 2017)

${ }^{4}$ For an introduction the relation between the FEP and Helmholtz' ideas, see (von Helmholtz (1868); Dayan et al. (1995))

${ }^{5}$ Note that Li Zehou also used Kant to compare Western and Chinese theories of the mind. He described the Kantian categories as a historical material-cultural "sedimentation"(Zehou and Samei, 2010)
} 
been formalized and specified, with root in statistical physics, to a level where it is compatible with scientific modeling and testing.

Friston' s theory holds that every process in the brain as well as self-organizing systems in general, can be seen as a process of PEM; that is, that the brain (partly) neuronally maintains a model of the world. Based on this model, it creates predictions about future input to its sensory systems. It then compares the actual sensory input to the predicted sensory input; the difference between which is the prediction error. It is this prediction error which the brain seeks to minimize, either through updating its model and corresponding predicted sensory input to better fit the actual input, or by acting upon the world to change the inputs to better fit predictions. It is then critical that our experience of the world is shaped not directly by our sensory input, but instead by our model and its predictions, which effectively turns around the process as described in classical cognitive science theories: instead of sensory input having higher cognitive processes as feedback, there is now a brain-wide predictive model which uses sensory input as feedback (Hohwy, 2013). Before I go into a more thorough description of this process, it bears to give a summary of the information-theoretical reasoning behind taking PEM to be a process important enough to found a grand theory of the brain, and more generally, life.

Probabilistically, existence can be defined as a system having a high probability of being in a relatively narrow set of possible states over time. Something does not recognizably exist if it is equally probable to be spread out over the universe as to be gathered in one place. Survival, then, necessarily corresponds to keeping that probability high - or, in informational-theoretic terms, to minimizing statistical surprisal over time (also known as entropy). Statistical surprisal is simply a measure for how improbable it is that a given system is in a given state: examples of high surprisal states would be a fish on land, a person in space without a spacesuit, or a cell with too much salt in it. By extension, life is a system that actively reduce surprisal. Any system at all which is selforganizing must reduce surprisal over time, be it a city, a cell or a social network, because if it does not, it would not exist (Friston, 2010).

One apparent issue with the account so far is that no system can actively reduce surprisal because no system can measure or access it directly. Knowledge of the surprisal of a state would require the impossible knowledge of all the infinite possible states that the system, and consequentially the universe, could be in. To proceed, we must first introduce the information theory term "free energy" from statistical thermodynamics. For the purpose of this paper, two conclusions of free energy' s application are relevant: under certain assumptions, minimizing prediction errors is equal to reducing free energy, and free energy is equal to the sum of statistical surprisal and the perceptual divergence between the system' s expectations of the world and the world' s actual states which can be written as free energy $=$ surprisal + perceptual divergence. This means that performing PEM either directly reduces statistical surprisal or puts a bound on it by reducing perceptual divergence by creating better models of the world (Friston, 2010; Hohwy, 2013). 
In a sense, this turns around the description of life and agency, much like how evolutionary theory did of our seemingly deliberately designed bodies. We do not reduce surprisal in order to survive; it is simply that the systems which reduce surprisal exist, and the ones that don' t do not, per definition. Culture, behavior, reproduction and homeostasis are all results of an emergent PEM process that is ultimately rooted in statistical physics.

\subsection{Perceiving the world}

The model kept by the system is a strictly statistical model, encoding probabilistic correlations between states of the world and between the world and sensory inputs to the system. The model is approximately Bayesian, which is a description of mathematically optimal updating of beliefs given new information. This model is always probabilistic and is continuously updated with the reception of new information. This updating happens on many timescales: almost instantaneous, low-level perceptual processes, higher, conceptual belief changes, and even on evolutionary time scales (Hohwy, 2013). As such, genetic, cultural, and cognitive processes are all ways to update the model and are not in a fundamental sense separate processes even though they are implemented very differently.

To infer about the world based on sensory inputs, the system needs to do a model inversion that is, it needs to reverse-engineer the set of causal probabilistic processes and world states that led to the given sensory input. For simple, linear processes this is easy, but the world is complex and nonlinear. Many different world states can give rise to the same sensory input, and many different sensory inputs can be related to the nearly-same world state. Therefore, the system' s model is hierarchical, with higher levels in the hierarchy encoding spatio-temporally general correlations (like the way light changes with seasons), while lower levels encode more specific correlations (like how the light depends on the specific time or place). The lowest levels are then correlations between world states and sensory input. Every level above these lowest levels makes predictions about the level below until the sensory input is received and prediction errors are propagated up through the hierarchy which then causes updating on different levels. Thus, every neuronal connection only does a simple, linear model inversion, in that they encode a prediction for only the level below. However, when this is distributed across the entire brain network, it can maintain a very complex model of the world that can support both generalizable and context specific models (Hohwy, 2013). The elegance of the theory is that it effectively bridges the gap between the mind and world without ever having direct contact with world states, and the system' s model still ends up carrying information about them. A very illustrative metaphor for this is Hohwy' s Leaky Dam Metaphor (Hohwy, 2013):

Imagine you are tasked with preventing water from getting through a leaky dam. The way the water leaks through the dam depends on various processes behind the dam, like water flows, rain, and seasonal changes, but you have no access to any of that - rather, you can only see where the water flows out of holes in the dam. In the beginning, you will do this rather frantically, running 
form leak to leak and stopping them manually, but slowly, you will start recognizing regularities in the way the water flows. For example, maybe every time a large flow of water comes from hole A, two small leaks are probable to follow from holes B and C, but only when hole E is dry. You then build a contraption, full of gears and levers, which automatically plugs holes according to the regularities you are witnessing. The regularities of the water flow change change over time, so the machine has to be continuously rebuilt to become more complex to take higher temporal scales into account. Eventually, the machine you have built will contain information of the processes on the other side of the dam, even if it never has any direct interactions with them. In the same way, the brain can carry information about the world the sensory veil. ${ }^{6}$ Of course, there are limits to how far this metaphor can stretch. For instance, it doesn' $t$ incorporate any option for deliberately sampling inputs from the world, which will be discussed later. It also assumes that there is an agent who does the predicting and the model building; while in the FEP framework, this is a distributed, unguided, and emergent process. The brain does not have a model, as much as it is a model of the world.

The hierarchical complexity of the brain' s model is thought to give an explanation of why our experience is so rich. Because our model contains many complex and rich correlations at the same time, we experience the world as such (Hohwy, 2013). It is also important to mention that the models of the brain must maintain simplicity because this prevents them from overfitting i.e. becoming overly specific to the current situation and available information. This also means that models will always have some error, so that they can be simplified enough to be generalizable. This means that the brain must also have predictions about when sensory inputs are reliable and worth using for model updating, and when they are rather consequences of simplified models. This is called precision weighting and is thought to be related to attentional processes (Brown et al., 2011; Hohwy, 2012). Additionally, it is thought to relate to pathological states like schizophrenia, where sensory input is deemed unrealistically reliable to the point that the brain maintains wrong models rather than updating them based on input. ${ }^{7}$ In the human case, parts of the model are implemented neuronally - but indeed, even genetics or cultural practices can be seen as predictions of the world, insofar as they prepare us for existing in a specific context. It is also important that all our predictions ultimately are based off of sensory inputs, which means that even abstract thought is grounded in our sensory-motor setup - that is, our body - from which our first-person perspective originates. The difference between humans and other organism is then not an absolute one, rather, we simply have more complex models, which contain both very specific and very general predictions. ${ }^{8}$

\footnotetext{
${ }^{6}$ The sensory veil can be described as a Markov blanket, a probabilistic definition of the system's boundaries. But note that system's will consist of many smaller Markov blankets (Ramstead et al., 2018); brain and body might be seen as simply different levels within this hierarchy

${ }^{7}$ Precision weighting is also thought to be related to dreams, where sensory input is suppressed by precision weighting because it is unreliable when the sensory apparatus isn't used - which leads models to be experienced without needing sensory input to sustain them (Hohwy, 2013)

${ }^{8}$ See (Pezzulo and Levin, 2018) for a generalization of the FEP to biological systems in general. Note that this brings to question how neurocentric versus embodied theories ought to be (Clark, 2017), and in which way the complex PEM processes in the brain
} 


\subsection{Active Inference}

So far, this account has left an impression of a strangely passive agent in the world who simply receives sensory input and continually creates better models of the world. But this does not explain the fact that humans seem to move and act, and neither does it seem to be able to reduce statistical surprisal, which was the entire reason PEM was brought in as a core mechanism. Perception may reduce free energy by creating better models of the world, which would correspond to lowering the perceptual divergence, and thereby putting a bound on surprisal - but if the system does not act on the world, then no matter how perfect the model, the system cannot keep itself from ending in high-surprisal states. This is where action comes in. There are, in general, two ways action can help PEM: it can help sample sensory inputs more optimally from the world and it can change the world in order to get the sensory input that is predicted. This is called active inference. The word is technically an oxymoron because the process is not an inference, but it helps highlight the very close relationship between action and perception of the world.

The first kind of active inference, sampling better inputs, would correspond to moving around an object to get a better look at it or to a scientist actively affecting the world in order to see what happens. In this way the brain can not only get information more efficiently, but it can also sample the specific inputs that can help it differentiate otherwise indifferentiable hypotheses by interfering directly with links in the causal chains that result in the sensory inputs. The second type is the one that can reduce surprisal, because it keeps the system in non-surprising states as opposed to simply modelling them better. It becomes clear how perception and action need each other, for it is not possible to act optimally on the world without good models of it, but a good model in itself is also not enough to keep existing. The balance and interplay between these two types of processes are critical, and a single, formal mechanism - PEM - allows that balance to be described and investigated numerically. Thus, not only perception and action, but also every related cognitive and behavioral phenomenon, seems describable in PEM terms in a way that links it intimately even with a probabilistic definition of life and existence (Hohwy, 2013).

Action, then, is not triggered by some command issued to the body. Rather, it is a prediction of where the body should be, which in order to avoid prediction errors makes the body moves to fit the prediction. The predictions about where the body will be then depends on predictions on higher levels - predictions that the system is not going to starve will, for example, cause the system to predict that it will eat, thereby predicting the movements of eating - which will cause the body to perform them as a PEM process. Action policies must also be structured hierarchically, with short duration actions like closing a hand at the lowest level, and longer timescales like climbing a mountain or establishing a company at higher levels. Formally, action occurs when the expected precision of the resulting states of an action is higher than that of the current states, and is thus a consequence of the entire model of the world, including the system itself. There needs to be good

differ from those of self-organizing organisms in general 
models, not just of how the world works, but how the body acts within it and how the sensory inputs react, in order to effectively predict outcomes of actions. Thus, the system needs to create a model of itself, including mental processes, and based on the many interoceptive sensory systems the body has of itself. Here might be an opening to describing how humans create an experience of self - although much more work needs to be done in this area (Hohwy, 2013).

Action and perception might both seem very much like a kind of self-fulfilling prophecy in this account. One could, for example, imagine that one sees something that looks like a face in a bush and that, in order to do PEM, one would attempt ad nauseam to cause sensory inputs that match that first wrong expectation, maybe ultimately by asking a friend to stick their head through the bush. Similarly, one might imagine a system simply updating its models in any number of absurd ways to account for surprising sensory input, maybe by imagining that the saltwater it is sinking into is simply an illusion. However, it must be remembered that the hypotheses generated by the brain are not arbitrary - they are constrained by the world - and that it often is much more PEM effective in the long-term to switch to perception or action, respectively, in those situations.

Another objection that has been made is that the PEM system seems to be fundamentally conservative. It is questioned why any such system would ever search out exciting or unforeseeable situations - indeed, why would any system not simply go into a room, shut the door and turn off the light in order to get as little input as possible? ${ }^{9}$ There are two answers to this charge. Firstly, PEM is done over time, and a system sitting in a dark room would not be able to uphold homeostasis for very long, thus resulting in a high amount of prediction errors in the future. Neither would such a system be robust to unexpected changes in the environment while those that explore and thereby create better models might fare better in general, and make up for the temporary predictions errors generated. This is especially important for humans who have complex models because of their complex social contexts. Additionally, humans do not expect themselves to only be in dark rooms. Rather, they expect themselves to be in very different states which always has sensory input and where some amount of prediction error is inevitable. The set of states that a system expects itself to be in is largely what determines it, and it is from these expectations that all sub-strategies and actions ultimately stem. Indeed, are all values and heuristics for action only effective as far as they reduce PEM relative to the phenotype and as such are dependent on the phenotype. A system with a phenotype that entails being found in dark rooms would indeed search for such states, but would also have to maintain such states over time, which may necessitate leaving the room at times, for example for getting food. Much work can be put into how these phenotypes differ between humans and other species as well as how they are generated, for example, through evolution (Hohwy, 2013). For some examples of the recent and varied applications of the FEP and active inference theories, see for example (Parr and Friston, 2017; Clark, 2017; Kaplan and Friston, 2018; Williams, 2018; Kirchhoff, 2018; Koelsch et al., 2019).

\footnotetext{
${ }^{9}$ thus coining this objection "the dark room problem"
} 


\subsection{Theory of Mind}

Although introducing the concept of Theory of Mind (ToM) is not necessary to understand free energy, it will be important for the discussion of Slingerland' s critique. ToM is the ability to infer mental states, beliefs, and emotions in others. As such, it is thought to be necessary for any complex, if not almost all, social processes. The term was originally used when trying to establish when this ability appeared developmentally in children (?Marvin et al., 1976), although it was later been questioned whether it is ToM that develops or simply general cognitive capabilities like working memory which develop enough to support such complex cognitive processes (Goldman et al., 2012). Within a free energy framework, ToM would be defined as simulating someone else' s thought processes and mental states simply as part of the model of the world, except it now includes modeling that someone else has a model of the world as well. This has been implemented in simple game contexts to investigate ToM in healthy and clinical human populations as well as animals (Diaconescu et al., 2014; Devaine et al., 2017; Khalvati et al., 2018; Colombo et al., 2018). It is important to say that inferring about the minds of others is not technically different from any other kind of model updating except that the model must be highly complex and have many subsets, and that models of own mental and bodily processes can be used as a starting point for predicting the other' s behaviour. It is important to mention that PEM systems can also use the behavior of other systems as feedback. For example, inferring about the world or even one' s own mental states is based on other' s reactions to own actions. There is great potential in this, but for it to be possible, the system needs to have good models of the others' s mental processes to use the feedback reliably. Indeed, the other systems also need to have good models of one' $\mathrm{s}$ own mental processes, so that they can give appropriate feedback. Social interactions on all scales thus become a complex process of updating own models as well as providing information for others to update their models which is an always unfinished, difficult, and ever-changing process. This signifies that the PEM processes in the FEP, although they happen within a self-organizing system, depends greatly on the social milieu they participate in and that even culture in general might be seen as part of, or a support for, the brain' s model of the world.

\section{Initial compatibilities}

There are several points on which Confucian theory seems to either agree straightforwardly with

the free energy principle. I will first give an account of those points; then I will move to the areas where the two frameworks either disagree or nuance each other.

\subsection{Knowledge serving action}

Both the free energy principle and the Confucian philosophy emphasize acting in the world rather than simply gathering knowledge about it. No matter how accurate, without being acted 
on or realized in the world, knowledge has no influence - it cannot minimize surprisal. Therefore knowledge, including its internal consistency, only matters insofar as it serves action. This also means that knowledge always becomes situated in a context where it can be used - in concrete predictions or actions. Additionally, knowledge cannot be separated from our body. It is intimately connected with our sensory apparatus and how we live and act in the world. Confucius' focus on dramatic rehearsal, rather than rule-following, also suits the free energy principle well. When compared to older implementations of artificial intelligence that simply followed a set of preordained rules that cold not account for every situation, free energy models perform ToM as a dynamic simulation of the effect of possible actions on other' s mental states. This simulation is exactly a dramatic rehearsal that allows to act appropriately in the situation.

\subsection{Becoming a PEM-effective human}

It seems that the free energy principle agrees with Mencius that humans naturally tend towards goodness. In a free energy framework, goodness or value depends on, or rather is defined as, effective PEM. Since the one core mechanism in humans and life is to perform PEM effectively, it is by definition true that they tend towards it although this is distinct from a teleological, innate or predetermined goodness, because PEM systems may fail in many ways. Live system will do good, but only insofar as they have good models of the world and themselves. This then is Mencius' human xing 性 that separates humans from animals - their capacity for more complex, flexible, and specific models that are needed for social interaction. They have the capacity to understand the changes in the world to a radically different degree than animals, which is why they can operate in such socially complex networks. But to do this, proper models on every hierarchical level need to be learned. This is indeed the point of the Confucian self-transformative project: to become, rather than innately be, a person who is able to participate in society in a way that is fruitful for both himself and others. This project must, to some degree, be the project of any human, for PEM depends on it. Exactly how this project is done is a harder question and also one that depends on the context the system is within. One insight from Mencius that seems describable in free energy terms would be the emphasis on internalizing values rather than following external requirements. This would correspond to updating models and action policies on higher levels of the model hierarchy, rather than lower ones, effecting changes in predictions and behavior that is spread out pervasively across situations rather than only in the context of external pressure. The Confucian sage cares for the root i.e. the uppermost model layers, which means that the desired actions can arise without an external trigger, and probably without much cognitive effort because they are already predicted and integrated in the general beliefs about the world. This also makes the action policies more robust to external pressure because it requires more input to update higher hierarchical models. Here lies perhaps a possible approach to developing a free energy principle definition of the spontaneity ziran 自然 of action that is applauded in Confucian thought.

page 13 of 22 


\subsection{A continous world in flux}

Confucianism and free energy also seem to agree upon a continuous world in constant flux. At least, any model layers except the highest order phenotypical ones are always changing and all parts of the model either directly or indirectly inform each other which would result in an experience of change and continuity. In a deeper sense a Bayesian model making predictions on even fundamental layers implicitly expects a changing world outside of experience too. If there were unchanging parts of that world, they would be learned eventually, making knowledge would the goal rather than prediction. But our constant need for updating models is better suited to a world that is always, and entirely, changing. Here it is also important that any model of the world - that is, any description, thought or even experience - is essentially an abstraction made based on sensory inputs, indeed paranomastic rather than definitory in nature. Categories are essentially always malleable sets of predictions rather than ontological descriptions which fits well with the Confucian insistence on continuous, holistic, impermanent world. At the same time, it must be stressed that while all models technically are malleable, many of them may not be in a practical sense. Some models are implemented genetically or culturally, and can only be updated on timescales longer than a human life. Some especially higher hierarchical models may be so suitable that they arise everywhere, for example models of mental processes without specific inclusion of neuronal (or bodily) subparts. It is a difficult to know exactly how many and to what degree there are models which are practically unchanging Additionally if is hard to identify these unchanging models as Platonic principles which are in sharp contrast to Confucian thought. In the free energy principle framework, they are not mutually exclusive because principles or unchanging "truths" are simply higher-level models that, although technically changeable, are practically held constant. Taken as general, higher-level concepts, Confucian ideas such as $l i$ 礼 become similar, except that they seem to have less concrete and specific predictions attached to them.

\subsection{Social interrelationality}

Finally, we We shall now see that the main virtues in Confucianism, ren 仁, yi 义, li 礼, zhi 智 and xin 信, suit well in a free energy framework because they are necessary for effective social interactions and therefore for PEM. Because the synchronous updating of other' $s$ and own models is such a complex process, it must depend heavily on $l i$ 礼. Because it constrains actions and roles within contexts, $l i$ 礼 reduces the uncertainty about the relation between mental states of others and their actions, enables more efficient predictions and thereby model updating. Indeed, it seems natural that no kind of complex social interaction, not to speak of societies on greater scales, is possible without some kind of $l i$ 礼 to guide actions and predictions of actions that allows proper modelling of other' s. This is very similar to how linguistic grammar must support and make possible linguistic communication.

page 14 of 22 
Similarly, xin 信 becomes directly supportive of PEM because it simplifies the model inversion greatly to be able to infer other mental states and intended actions directly from their claims. Zhi 智 seems to almost directly denote the need for good models of the world, others and the self in general, to be able act in any context, especially social contexts. In contrast, yi 义 seems to describe the need for context-sensitivity, and therefore context modeling, to underlie actions so as to make them appropriate in specific contexts. Lastly, ren 仁 seem to denote clearly the complex interplay between social interaction and good models of the world in general by spelling out that it is necessary to take part effectively in social contexts to allow for the feedback that is so critical for updating models as complex as those of humans. Proper co-humanity, Confucianism seems to say to free energy, is critical for proper PEM. This means that a social PEM system becomes, in an utterly intimate way, dependent on a functioning society around them on all levels. There has not yet been much focus on this aspect within Free Energy though, and it seems a fruitful aim to begin modelling the complex processes where humans partake in shaping each-others models and actions - that is, creating each other in the Confucian sense of the word. ${ }^{10}$ A possible extension of this approach would be to also model PEM systems as nested within each other. The current research in the field has originally focused on single systems, but makes the point that cells and cities can be described as PEM systems as well. In this hierarchically interwoven set of systems - cells making up bodies which make up overlapping social groups - each sub-system depends critically on the efficiency of the PEM processes of both the sub-systems it consists of, and those higher larger systems which it partakes in. ${ }^{11}$ Indeed, this is ultimately to the extent where the entirety of the cosmos that makes up the human social world depend on every subpart of it performing PEM effectively. In this sense, man is continuous with tian 天, and can, with good enough models nested within him both in brain/body and socio-cultural context, affect it to possibly limitless degrees - develop the flood-like $q i$ 气 that Mencius describes. Much work is needed to approach this formally, and to be able to describe it more concretely and nuanced. However, inspired by Confucianism, modelling human PEM systems within a network of other systems seems an obvious direction to go to be able to understand the human system in a social context.

\section{Disagreement and complementarity}

As previously stated, Confucianism can inspire and guide work in the free energy principle. Additionally, the free energy principle might be able to formalize and specify Confucian ideas. Here, I will go into areas where it is less clear whether the two frameworks can agree and which directions of research this points towards. There are two related, main points: conscious experience and religious value and enchantment compared to the reductionist, materialist framework of free energy.

\footnotetext{
${ }^{10} \mathrm{~A}$ recent article by Veissière et al. (2019) has indeed attempted to describe inter-personal co-creation in free energy terms

${ }^{11}$ Describing this as nested Markov blankets has been suggested in (Kirchhoff et al., 2018)
} 


\subsection{A question of consciousness}

Hohwy makes clear that the free energy principle does not solve the hard problem of consciousness (Hohwy, 2013). In other words, it cannot describe how neuronal-mathematical mechanisms can result in a conscious experience of the world. Therefore, there is, at least inherently within the theory, no such thing as an experience of the world, or of choice, which technically leaves humans as " dead" , algorithm-based processes without a will or values separate from a statistical-physical automatic process. Superficially, this seems in very stark contrast with Confucianism, which is a personal project that involves acting upon our experience of the world in order to improve it and out experience of it. This seems to be the same conflict that arises between most humanistic and natural, scientific theories of the human mind and is perhaps one that, because it stems from the very nature of the different approaches, cannot or should not be attempted to be solved. Another, and almost opposite, conflict also seems to arise from the fact that the free energy brain only experiences models of the world, which, although they are not arbitrarily related, are ultimately different from the true world states. This seems to introduce the appearance/reality dichotomy which Confucianism never subscribed to. I will suggest here that both of these conflicts might be addressed in one argument, although to differing degrees. Firstly, the dichotomy between the experienced and the real world is dispelled by the fact that free energy is a materialist theory. There is no mind separate from the body which contains experiences. The experience is instantiated in neuronal firing and possibly in other structures making up the hierarchical model. The experience is indeed part of the world on par with any other process that is part of the world, but at the same time first-person perspectives do exist: the world as modelled by the brain is different within each individual brain and PEM system. ${ }^{12}$ Since perspectives are models, and an entire system makes up its model, it is correct to say that these perspectives make up what is single persons in the world, and that they are absolutely unique but also subparts of the process of the world. Here the terminology of perspectives being foci within the larger field ${ }^{13}$ can be applied without friction. These foci also contain within them the whole field because they, quite literally, model it and are shaped intimately by it. And this quickly brings to mind that people are already described in the Yijing as models of the cosmos. Each PEM system is continuous with the world through their sensory apparatus and action on the world but from the unique perspective that make up who they are. This still leaves the notorious question of why there is an experience at all. If minds are simply ongoing neuro-statistical processes, why do we experience the world? I do not think this can be answered here, but perhaps a new approach that can be offered by Confucianism is to see experience and material as part of a processual qi 气 cosmology. $Q i$ 气 as constitutional, processual, relational energy can denote both a rock lying still, a movement of the arm, or an ongoing modelling of the world because all of those are relational

\footnotetext{
${ }^{12}$ See also a recent article by Solms (2019), phrasing mental and physical appearances as different complementary perspectives on the same reality, (dual-aspect monism), and integrating his view with the free energy principle

${ }^{13}$ A terminology often used by Ames (Ames, 2011)
} 
processes. This is indeed the difference between simply a set of neurons firing and a set of neurons firing in exactly the way that makes up the specific mind. There is not a difference in material, nor only in action, but in $q i$ 气 which is the intersection between those two things. This account is not exhaustive, for it is still necessary to find out what exactly is the difference between a process where experience occurs and one where it does not as well as many details thereafter. The $q i$ 气 worldview of the ancient Chinese does not solve the problem of consciousness on its own, at least with regards to modern cognitive science, but it may provide a vocabulary better suited for describing the issue which can lead to the possibility of a solution. Related here, I would like to shortly mention the perhaps parallel work of Donald Crosby' s work on what he calls radical materialism. His work is a redefinition of material as not substance but process: matter is what matter does. He claims that, in addition to be more suited to modern physics, it can also be an opening for untangling the issues of consciousness, free will and spirituality (Crosby, 2016). I don't think Crosby's work can yet propose concrete solutions to these issues, but it seems a strong possibility that his work might be able to communicate with the two frameworks discussed here. Additionally, there has already been work proposing compatibility between enactivist theories and classical pragmatism (?), the latter of which has already been related to Ames' Confucianism and the $q i$ 气 cosmology, suggesting pragmatism as another mediator between cognitive science and classical Chinese philosophy on the question of consciousness. There is of course no kind of certainty with this question. Even if frameworks such as those previously mentioned were to further the debate, it is likely that more challenges are waiting around the corner. However, it does seem a promising path forward in the landscape of cognitive science where fruitful approaches to the question of consciousness are notoriously sparse.

\subsection{Science and life}

As a final note on the comparison between the two theoretical frameworks, it should be mentioned that, even if they can be conceptually commensurable, the two frameworks have vastly different emphases. Confucian theory is largely meant to inspire a way of living and of improving society and of making human lives valuable and enchanted while the free energy principle is specifically aimed at creating accurate descriptions of how the mind and brain work. While I do not think it is impossible to use the details of the free energy principle, at least metaphorically, in daily life, the scientific theory is not particularly specialized in being implementable in normal social situations.

Of course, there is a vast amount of possible applications of this knowledge in fields from artificial intelligence to therapy. However, these are still applications of a scientific theory which, in the first case, is trying to create a good mathematical description of the world. Interestingly, this echoes the two functions of the PEM system. While the scientific theory is about creating better models about the world, Confucian theory is about acting in the world; however, both of those goals depend greatly on each other. Related to this, it is noteworthy that both of the theories prioritize action over knowledge. This means that knowledge and thought does not necessarily have to be correct

page 17 of 22 
to any higher degree than what is useful. This brings to mind the notorious saying about statistical models attributed to George Box: "All models are wrong, but some are useful" . According to the free energy principle, none of our models, and therefore, none of our experiences, beliefs or philosophies, are completely true, but they might still be useful for living in the world. Therefore, it is perhaps less important that theories like Confucianism and the free energy principle are able to be completely unified conceptually, as long as they can fruitfully inspire and direct each other and society. Neither theory seems to be able to be reduced to the either or entirely encapsulate its points. Free energy has little to give in terms of enchanting daily life, but Confucianism does not provide formal descriptions, for example. But it still seems a conversation between the theories can be useful,and that is perhaps all that ultimately matters.

\subsection{An answer to Slingerland}

In the above I attempt to show how a prominent, although controversial, framework within cognitive science often seems to be fluently compatible with Ames' Confucianism, and that in the areas where it is not immediately compatible, the two frameworks may be of value to each other. This, in broad terms, indicates a rebuttal of Slingerland' s claims that Ames' Confucianism is incompatible with modern cognitive science. For this to be conclusive, a thorough comparison would need to be done, for the framework might integrate differently with Slingerland' s and Ames' standpoints on different parts of the discussion. Such a complete examination is beyond the scope of this paper, but there is room to make an examination of a Slingerland' s core argument of the impossibility of a mind/body holism. Here, the free energy principle might provide a framework that can alleviate that claimed tension between cognitive science and Ames' Confucianism.

Slingerland claims that the persistent phenomenon of Theory of Mind (ToM) (see chapter 3) must mean that there universally are different conceptions of mind and body. He says that a weak kind of Cartesian dualism must always be present, that is, mind and body can only be seen as separate entities with some kind of overlap. Accordingly, the strong holism which he attributes to Ames is impossible. Taking ToM as a fundamental and universal process in humans, it is not sound to claim that the Chinese saw no difference between mind and body. Slingerland raises an excellent point that we should avoid forgetting all that we have in common despite cultural changes, however fundamental. In contrast, it is a risk to interpret scientific findings within one cultural context and not consider how they could be interpreted within another. I think the free energy principle' $\mathrm{s}$ conception of ToM is a good example of a conception that, if anything, seems to fit a weak, holistic description better. Here, the mental processes are abstractions of processes that are essentially as biological as any other bodily process. The conception of mental states without reference to body is useful because they summarize complex processes. It is a simplification of the model of the other agent which creates the experience of a mind separate from body. This does not fit with (weak) Cartesian dualism, though, for there is not absolute substances like a mind and a body that overlap.

page 18 of 22 
Rather, there is a coherent whole, a true world state which we have no direct access to, but which we model by abstracting out such processes as mind and body. Behuniak claims in his review that Slingerland' s critique is unfounded because Ames' standpoint, a kind of weak holism, would be essentially equivalent to Slingerland' s weak dualism (Behuniak, 2019b). However, there seems to be a difference in whether mind and body are two abstractions out of a whole or whether they are two separate entities which overlap. I think the first can have as much, if not even more, claim to fitting modern insights from cognitive science. Of course, the free energy principle is not universally accepted in its field yet, but the fact that such a reasonable theory can be so compatible with Ames' Confucianism means that it is not substantiated to claim that the best insights from cognitive science disagree with Ames' claims.

Of course, it might be interjected that Mencius and Confucius didn' $t$ know any theory like the free energy principle, and that perhaps they were not able to realize that their own conceptions of mind and body were models of the world rather than ontological substances. Maybe we are overinterpreting the ancient philosophers based on the scientific and philosophical progress in the two millennia since their time. And even if they were able to conceptualize a holistic cosmos, it is not certain that their views represented the general mindset in ancient China. Indeed, the free energy would claim, our biological underpinnings shape us, for they make up the higher hierarchies of our mental-bodily models and priors and shape our sensor input. However, the way our body and world is modelled, and therefore described, thought of, and experienced, is heavily shaped by culture. There does not seem to be any specific reason grounded in cognitive science that the ancient Chinese were unable to model the world as a holistic unity from which mind and body are abstracted.

I do have faith in the philosophical capabilities of current and ancient scholars to produce descriptions of the world which does not exclude the Amesian account of the classical Chinese worldview, especially given that it fits so well with insights from modern cognitive science.

\section{Conclusion}

In this paper, I have given an overview of the free energy principle, a new and ambitious grand theory of the mind becoming gradually more popular within cognitive science and related fields. I have also given a brief overview of the Confucian worldview and transformative project, built on the work by Roger Ames. Comparing these two widely different theoretical frameworks, I find that they are on many points immediately compatible. The Confucian theory can be re-described in and be supported by the free energy principle. There are also some areas where they seem to add onto each other more fundamentally. Confucianism would suggest that work on the inter-relationality between PEM systems is critical and that it is important to work more on how they must be nested within each other. It also might supply a different vocabulary for entering the debate about conscious experience within reductionist materialist frameworks. On the other hand, the free energy principle might allow nuancing of Confucian ideas, for example by being able to describe them numerically 
in order to test them against empirical evidence and allow for a discussion with other cognitive science-based standpoints such as Slingerland' s critique of Ames' interpretation. All in all, it is exciting to see how seamlessly these two frameworks, coming from widely different times, cultures and academic traditions, can communicate. It brings hope that in the future, as scientific theories of the human mind get broader, only more of this type of collaboration can take place, hopefully providing new insights within many different academic areas.

\section{References}

Ames, R. T. (2011). Confucian role ethics : a vocabulary. The Chinese University Press.

Behuniak, J. (2019a). Response to Edward Slingerland. Dao, 18(3):489-491.

Behuniak, J. (2019b). Slingerland, Edward, Mind and Body in Early China: Beyond Orientalism and the Myth of Holism. Dao, 18(2):305-312.

Bogacz, R. (2017). A tutorial on the free-energy framework for modelling perception and learning. Journal of Mathematical Psychology, 76.

Brown, H., Friston, K., and Bestmann, S. (2011). Active inference, attention, and motor preparation. Frontiers in Psychology.

Buckley, C. L., Kim, C. S., McGregor, S., and Seth, A. K. (2017). The free energy principle for action and perception: A mathematical review.

Clark, A. (2017). Busting Out: Predictive Brains, Embodied Minds, and the Puzzle of the Evidentiary Veil. Nous, 51(4).

Colombo, M., Elkin, L., and Hartmann, S. (2018). Being Realist about Bayes, and the Predictive Processing Theory of Mind.

Colombo, M. and Wright, C. (2017). Explanatory pluralism: An unrewarding prediction error for free energy theorists. Brain and Cognition, 112:3-12.

Crosby, D. A. (2016). Probabilism, Emergentism, and Pluralism: A Naturalistic Metaphysics of Radical Materialism. Technical Report 3.

Dayan, P., Hinton, G. E., Neal, R. M., and Zemel, R. S. (1995). The Helmholtz Machine. Neural Computation, 7(5):889-904.

Devaine, M., San-Galli, A., Trapanese, C., Bardino, G., Hano, C., Saint Jalme, M., Bouret, S., Masi, S., and Daunizeau, J. (2017). Reading wild minds: A computational assay of Theory of Mind sophistication across seven primate species. PLOS Computational Biology, 13(11):e1005833. 
Diaconescu, A. O., Mathys, C., Weber, L. A. E., Daunizeau, J., Kasper, L., Lomakina, E. I., Fehr, E., and Stephan, K. E. (2014). Inferring on the Intentions of Others by Hierarchical Bayesian Learning. PLoS Computational Biology, 10(9):e1003810.

Engel, A. K., Friston, K. J., and Kragic, D. (2016). The Pragmatic Turn : Toward Action-Oriented Views in Cognitive Science. DONE, (March 2017):1-15.

Friston, K. (2010). The free-energy principle: a unified brain theory? Nature Reviews Neuroscience, 11(2):127-138.

Friston, K., Kilner, J., and Harrison, L. (2006). A free energy principle for the brain.

Friston, K. J. and Stephan, K. E. (2007). Free-energy and the brain. Synthese, 159(3):417-458.

Goldman, A. I., Margolis, E., Samuels, R., and Stich, S. (2012). Theory of Mind Oxford Handbook of Philosophy and Cognitive Science (2012) Edited by. Oxford Handbook of Philosophy and Cognitive Science, pages 1-25.

Hohwy, J. (2012). Attention and Conscious Perception in the Hypothesis Testing Brain. Frontiers in Psychology, 3:96.

Hohwy, J. (2013). The predictive mind. Oxford University Press.

Kaplan, R. and Friston, K. J. (2018). Planning and navigation as active inference. Biological Cybernetics, 112(4).

Khalvati, K., Park, S. A., Mirbagheri, S., Philippe, R., Sestito, M., Dreher, J.-C., and Rao, R. P. N. (2018). Bayesian Inference of Other Minds Explains Human Choices in Group Decision Making. bioRxiv, page 419515.

Kirchhoff, M., Parr, T., Palacios, E., Friston, K., and Kiverstein, J. (2018). The markov blankets of life: Autonomy, active inference and the free energy principle. Journal of the Royal Society Interface, 15(138).

Kirchhoff, M. D. (2018). Autopoiesis, free energy, and the life-mind continuity thesis. Synthese, 195(6).

Koelsch, S., Vuust, P., and Friston, K. (2019). Predictive Processes and the Peculiar Case of Music.

Marvin, R. S., Greenberg, M. T., and Mossler, D. G. (1976). The Early Development of Conceptual Perspective Taking: Distinguishing among Multiple Perspectives. Child Development, 47(2):511.

Parr, T. and Friston, K. J. (2017). Working memory, attention, and salience in active inference. Scientific Reports, 7(1). 
Pezzulo, G. and Levin, M. (2018). Embodying Markov blankets: Comment on "Answering Schrödinger's question: A free-energy formulation" by Maxwell James Désormeau Ramstead et al.

Ramstead, M. J. D., Badcock, P. B., and Friston, K. J. (2018). Answering Schrödinger's question: A free-energy formulation. Physics of life reviews, 24.

Slingerland, E. (2013). Body and mind in early China: An integrated humanities-science approach. Journal of the American Academy of Religion, 81(1):6-55.

Slingerland, E. (2019a). Mind and Body in Early China. Oxford University Press.

Slingerland, E. (2019b). Response to Jim Behuniak. Dao, 18(3):485-488.

Slingerland, E. and Chudek, M. (2011). The Prevalence of Mind-Body Dualism in Early China. Cognitive Science, 35(5):997-1007.

Solms, M. (2019). The hard problem of consciousness and the free energy principle. Frontiers in Psychology, 9(JAN).

Veissière, S. P. L., Constant, A., Ramstead, M. J. D., Friston, K. J., and Kirmayer, L. J. (2019). Thinking Through Other Minds: A Variational Approach to Cognition and Culture. Behavioral and Brain Sciences, pages 1-97.

von Helmholtz, H. (1868). Über das Sehen des Menschen. In Vortäge und Reden 1, pages 265-366. GRIN Verlag GmbH.

Williams, D. (2018). Predictive coding and thought.

Zehou, L. and Samei, M. B. (2010). The Chinese aesthetic tradition. University of Hawai i Press. 